\title{
Original
}

\section{miR-27b-3p Was Involved in Retinoic Acid-induced Abnormal Early Myogenic Differentiation of C2C12 Cells via Targeting CaMKII}

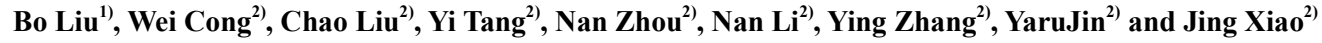 \\ ${ }^{1)}$ Institute of Genome Engineered Animal Models for Human Diseases, Dalian Medical University, Dalian, China \\ 2) Department of Oral Pathology, College of Stomatology, Dalian Medical University, Dalian, China \\ (Accepted for publication, April 3, 2018)
}

\begin{abstract}
Myogenic differentiation is an important stage within the multi-step process of skeletal muscle development. We previously found that excess retinoic acid (RA) could induce derangement of myofilaments in the embryonic tongue by inhibiting differentiation through $\mathrm{Wnt5a} / \mathrm{CaMKII} \delta$ (calcium/calmodulin-dependent protein kinase II delta) pathway. Furthermore, our recently studies indicated that excess RA can directly induce abnormal expression of series of miRNAs in embryonic tongue, including miR-27b-3p. miR-27b-3p has been reported as a regulator involved in kinds of tumors development. In addition, miR-27 was proved to negatively regulate adipogenesis. It has been indicated that miR-27b could down-regulate the expression of Pax 3 and Pax7 and thus inhibits myoblast proliferation. Here, we found that the expression of miR-27b-3p increased at early stage of myogenic differentiation (differentiation day 2, D2) in RA-treated $\mathrm{C} 2 \mathrm{C} 12$ cells, but $\mathrm{CaMKII} \delta$ expression was reduced. Furthermore, bioinformatics analysis predicted that miR-27b-3p targets the 3'UTR of CaMKII $\delta$. The direct interaction between of miR-27b-3p and CaMKII $\delta$ was confirmed by luciferase reporter assays. More importantly, rescue experiments indicated that CaMKII $\delta$ mediated miR-27b-3p to regulate the early differentiation defects induced by excess RA, and which was achieved mainly via Myogenin. In conclusion, excess RA disturbed the early differentiation of $\mathrm{C} 2 \mathrm{C} 12$ cells by stimulating miR-27b-3p expression which targeted CaMKII $\delta$, and then controlled the expression of Myogenin, those above implying new mechanism in myogenic differentiation and miR$27 \mathrm{~b}-3 \mathrm{p}$ may act as a new biomarker for muscle disease.
\end{abstract}

Key words: Retinoic acid, miR-27b-3p, CaMKII $\delta$, Myogenic differentiation

\section{Introduction}

Vertebrate skeletal muscle development is a complex and multistep process involving the myoblasts withdrawal from cell cycle and differentiation into multinucleated myotubes and myofibers terminally. Among which, myoblast differentiation is considered as the most crucial step for determination of muscle cell fate and final maturation of myofiber. Genes govern the myoblast differentiation in a spatiotemporal manner, which mainly including myogenic regulatory factors (MRFs), MEF2 and SRF ${ }^{1}$. Any variation of the factors involved in this process would lead to muscle related disease.

As an oxidative metabolite of vitamin A, physiological doses alltrans retinoic acid (RA) is essential for normal vertebrate embryonic development. RA deficiency or excess is teratogenic in nearly all vertebrate species, and could results in various degrees of congenital defects $^{2}$. Our previous study about the palatal development showed that certain intragastric dose of RA on 10th day of pregnancy would result in fetal cleft palate mouse by inhibiting the growth of palatal mesenchymal cells ${ }^{3)}$. Furthermore, we found that the RA induced cleft palate fetal mouse was accompanied by tongue malformation, because of the disruption of proliferation and differentiation of tongue muscle. More importantly, we found that wnt5a/calcium/calmodulin-dependent protein kinase II delta (CaMKII $\delta$ ) pathway was closely related with this process.

microRNAs (miRNAs) are about $22 \mathrm{nt}$ single-stranded non-coding RNAs, which function to promote mRNA degradation or inhibit translation by biding the seed sequences of their target mRNAs ${ }^{4}$. Correspondence to: Dr. Jing Xiao, Department of Oral Pathology, College of Stomatology, Dalian Medical University, 9 Western Section, Dalian, 116044 , China; Tel: +86-0411-86110218; Fax: +86-0411-86110219; E-mail: xiaoj@ dlmedu.edu.cn.
miRNAs express in a tissue-specific manner ${ }^{5}$ and those miRNAs riched in both human and mouse heart and skeletal muscle ${ }^{6}$ were known as myomiRs, mainly including miR-1, -133a and -206. These myomiRs were proven to be highly and specifically expressed during the myogenic differentiation process and to be regulated by MFRs and other myogenic transcriptional factors, and which return target commonly differentiation-related genes ${ }^{7}$. In addition, many non-myomiRs were also proved to be involved in myogenic differentiation process by interacting with the myogenic differentiation-related genes, including miR-31, $-34,-22,-145$ and so on ${ }^{8}$. The aberrant expression of these miRNAs could subsequently lead to different muscle malformation or diseases.

miR-27b has been proven to inhibit adipocyte differentiation and be involved in various tumors development ${ }^{9}$. Actually, the role of miR$27 \mathrm{~b}-3 \mathrm{p}$ in myogenesis is controversial. Crist et al proved that miR$27 \mathrm{~b}$ could inhibit myoblast proliferation and improve differentiation by down-regulating Pax3/7 expression ${ }^{10}$. The other study indicated that miR-27b could inhibit the expression of myostatin and thus improve the proliferation of myoblasts ${ }^{11)}$. In addition, miR-27b directly targeted Gai2 and then inhibited the proliferation and differentiation of satellite cells ${ }^{12)}$. Taken together, the role of miR-27b in myogenesis needs further exploration and the expression and mechanism of miR-27b in RA induced abnormal myogenic differentiation remain uncovered.

In this paper, we showed the increase of miR-27b-3p expression in RA induced abnormal myogenic differentiation at early stage. Functionally, gain- and loss-of function experiments indicated that miR$27 b-3 p$ inhibited the early differentiation of myoblasts. Furthermore, we identified that CaMKII $\delta$ was a downstream target of miR-27b$3 p$. Finally, rescue experiments determined that CaMKII $\delta$ mediated 
J.Hard Tissue Biology Vol. 27(3): 173-180, 2018

Table 1. RNA oligonucleotides' sequences used in this study

\begin{tabular}{l|l}
\hline \multicolumn{1}{c|}{ Oligonucleotide } & Sequence (5'-3') \\
\hline miR-27b-3p mimic & UUCACAGUGGCUAAGUUCUGC \\
& AGAACUUAGCCACUGUGAAUU \\
Duplex NC & UUCUCCGAACGUGUCACGUTT \\
& ACGUGACACGUUCGGAGAATT \\
miR-27b-3p inhibitor & GCAGAACUUAGCCACUGUGAA \\
Inhibitor NC & CAGUACUUUUGUGUAGUACAA \\
siCaMKIIס & GCUAGAAUCUGCCGUCUCUTT \\
siNC & AGAGACGGCAGAUUCUAGCTT \\
& UUCUCCGAACGUGUCACGUTT \\
& ACGUGACACGUUCGGAGAATT
\end{tabular}

Table 3. qRT-PCR Primer and Sequences

\begin{tabular}{ll|l}
\hline Primers & Accession NO. & \multicolumn{1}{|c}{ Sequnce(5'-3') } \\
\hline MyoD & NM_010866.2 & F: CGCTCCAACTGCTCTGATGGCA \\
& & R:TGCTGCTGCAGTCGATCTCTCA \\
Myogenin NM_031189.2 & F: CCAGTGAATGCAACTCCCACA \\
& R: ATGGACGTAAGGGAGTGCAGATT \\
CaMKIIS & NM_001025438.1 & F: AGAAGTTCAAGGCGACCAGCA \\
& & R:GGGTATCCCACCAGCAAGATGTAG \\
GAPDH & NM_008084.2 & F: TGTGTCCGTCGTGGATCTGA \\
& & R: TTGCTGTTGAAGTCGCAGGAG \\
\hline
\end{tabular}

the action of miR-27b-3p in RA induced early abnormal myogenic differentiation. Given the above, we found that miR-27b-3p, a novel regulator for muscle formation, inhibits myoblasts differentiation by directly targeting CaMKII $\delta$.

\section{Cell culture}

\section{Materials and Methods}

Both C2C12 and 293Tcell lines were purchased from the Cell Bank of Type Culture Collection of Chinese Academy of Science (Shanghai, China). Cells were cultured in high-glucose Dulbecco's modified Eagle's medium (DMEM) Invitrogen, Carlsbad, CA, USAsupplemented with $10 \%$ fetal bovine serum (FBS) (Gibco by Invitrogen) at $37^{\circ} \mathrm{C}$, $5 \% \mathrm{CO}_{2}$ in a humidified incubator. For inducement of myogenic differentiation, growth medium (GM) with $10 \%$ FBS was changed to high-glucose DMEM containing 2\% horse serum (Hyclone, Logan, UT, USA) when cells reached $80 \%-90 \%$ confluence. The culture medium was refreshed every 2 days and RA (Sigma, St. Louis, MO, USA) supplement in the culture medium was prepared as we previously described ${ }^{13)}$.

\section{Cell transfection}

The procedure of RNA oligonucleotides transfection was carried out as described in our previous study ${ }^{14)}$. The RNA oligonucleotides' sequences are listed in Table 1.

\section{Dual-luciferase reporter assay}

For the luciferase reporter assay, pmirGLO-CaMKII $\delta$-3'UTR wild type (WT) and pmirGLO-CaMKII $\delta$-3'UTR mutant (Mut) were purchased from GenePharma Co., Ltd (Shanghai, China). The oligonucleotides' sequences are described in Table 2. 293T cells were seeded in 96 well plates. After incubation at $37^{\circ} \mathrm{C}$ overnight, cells were transfected with miR-27b-3p, miR-140-3p or Duplex NC, and cotransfected with pmirGLO-CaMKII $\delta$-WT or pmirGLO-CaMKII $\delta$-Mut using Lipofectamine 3000 reagent (Invitrogen, USA). Following 24 hours transfection, the luciferase activities were detected using Dual-
Table 2. The oligonucleotides' sequences used in this study

\begin{tabular}{l|l}
\hline Oligonucleotide & \multicolumn{1}{|c}{ Sequence (5'-3') } \\
\hline pmirGLO- & TATTGAGCCGTGTAACCTGCCAATGAG \\
CaMKII $\delta$-WT & ATCTGTGAACCTGCCAATGA \\
& GATCTGTGAACC TGCCAATGAGATCTG \\
& TGAAAAAGTAATGGCCTCA \\
\hline pmirGLO- & TATTGAGCCGTGTAACCTGCCAATGAG \\
CaMKII $\delta$-Mut & ATTTCACAGCCTGCCAATGA \\
& GATTTCACAGCCTGCCAATGAGATTTC \\
& ACAGAAAGTAATGGCCTCA \\
\hline
\end{tabular}

Luciferase Reporter Assay System (Promega, Madison, WI, USA) following the manufacture's description.

\section{Quantitative real-time PCR}

Total RNA extraction from cells was carried out with RNAiso Plus reagent (Takara Bio Inc., Otsu, Japan). To detect the expression of miR27b-3p reverse transcription was done using a High-Capacity cDNA Reverse Transcription Kit (4366596, Applied Biosystems, Foster City, CA, USA) with specific miRNA primers (000409, ABI), and U6 was used for the internal control (0001973, ABI). Q-PCR was followed on ABI 7500 Fast Real-Time PCR System with the TaqMan ${ }^{\circledR}$ Universal Master Mix II Kit (no UNG, 4440040, ABI). cDNA was synthesized by using PrimeScriptTM RT reagent Kit (Takara) followed by Q-PCR with the SYBR Premix Ex TaqTM Kit (Takara) on a Thermal Cycler DiceTM Real Time System (TP800, Takara) with GAPDH as an internal control. The sequences of the primers were listed in Table 3 .

\section{Immunocytochemistry}

Cells cultured in 24 well plates were fixed with $4 \%$ paraformaldehyde (PFA) for $30 \mathrm{~min}$ at room temperature and permeabilized with $0.5 \%$ Triton-X100 (Solabio, Beijing, China). After incubated with primary antibody against mouse myosin (1:50, Maixin, Fuzhou, China) or antibody against CaMKII $\delta\left(1: 200\right.$, Abcam, Cambridge, UK) at $4^{\circ} \mathrm{C}$ overnight, cells were blocked with fluorescent secondary antibody (1:200, goat anti-mouse, DyLight 488, or goat anti-rabbit, Alexa Fluor 594, a11037, Invitrogen) for 1 hour at room temperature. The nuclei were stained by DAPI (D9542, Sigma) for 10 min. Finally, a fluorescence microscope (DP72, Olympus, Tokyo, Japan) was used to capture the pictures from at least three regions in each well.

\section{Western blot}

Cells were harvest and lysed in ice-cold RIPA buffer for $30 \mathrm{~min}$ on ice. The concentration of total protein was determined by a BCA protein assay kit (Boster, Wuhan, China). Equal amounts of protein were separated by $5 \%$ stocking and $12 \%$ running SDS-PAGE (Bio-Rad, Hercules, USA) and subsequently transferred to PVDF membranes (Bio-Rad). After the membranes were blocked with Tris-buffered salineTween-20 (TBST) containing 5\% non-fat milk for 2 hours at room temperature, the membranes were incubated with the primary antibodies against CaMKII (ab105502, Abcam), myosin (05-716, Millipore, Billerica, MA, USA) and GAPDH (Santa Cruz Biotechnology, Inc, Santa Cruz, Calif, USA at $4^{\circ} \mathrm{C}$ overnight. After washed three times using TBST, the membranes were incubated with secondary antibodies for 1 hour at room temperature. The protein bands were detected by the enhanced chemiluminescence substrate (Boster).

\section{Statistical analysis}

All presented results were collected from at least three independent experiments. The differences between groups were analyzed by two- 
Bo Liu et al.: miR-27b-3p Targeting CaMKIII Rescued Excess RA Induced Abnormal Myogenic Differentiation

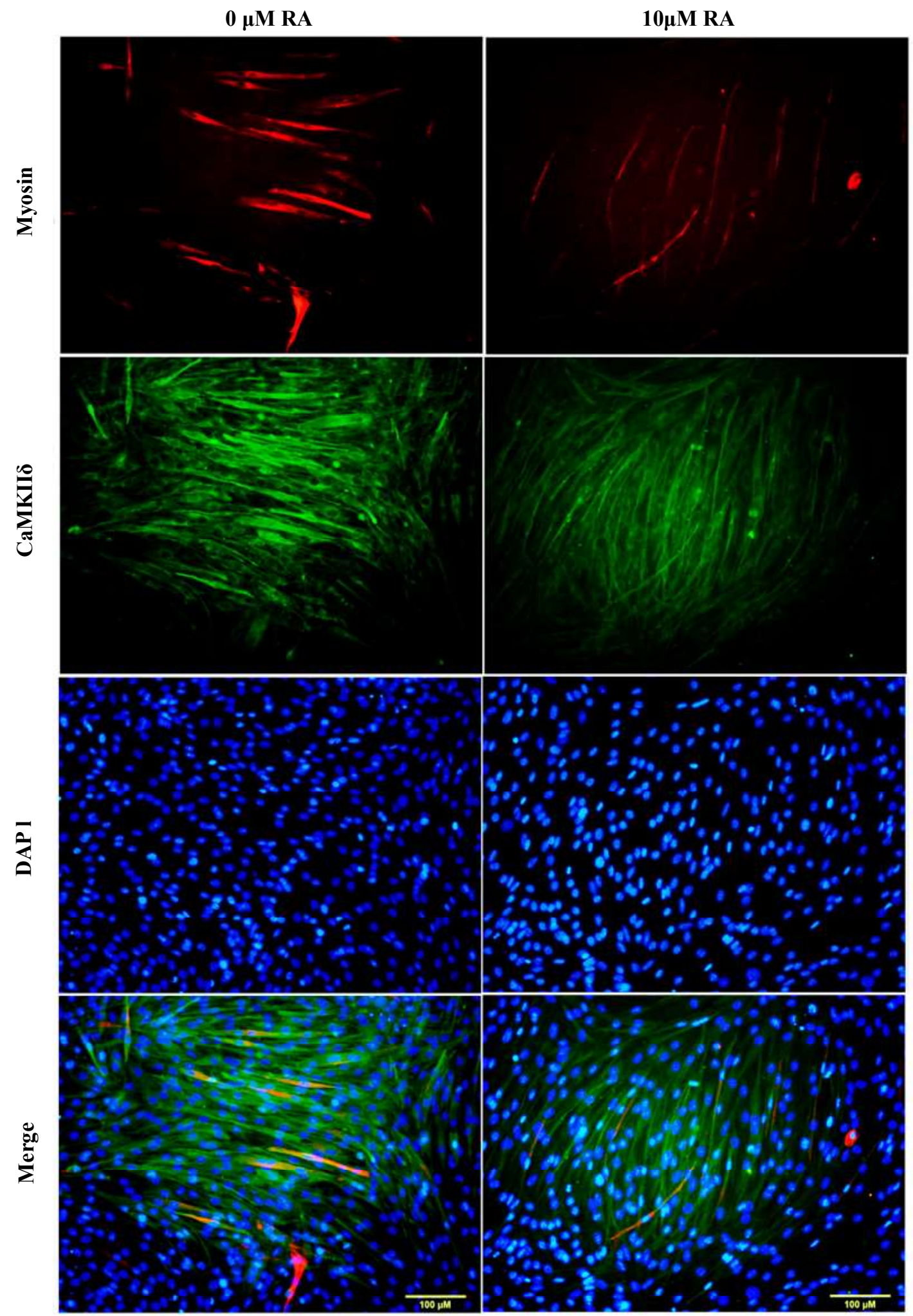

Figure 1. Immunofluorenscence staining analyzed the expression of myosin and CaMKII $\delta$ in cells cultured in DM supplemented with or without $10 \mu \mathrm{M}$ RA for 2 days. $($ Bar $=100 \mu \mathrm{m})$

tailed Student's t-test using SPSS 17.0 software (SPSS Inc., Chicago, IL, USA) and the statistical consequences were shown as the means \pm SD. P-value $<0.05$ was considered to be statistically significant.

\section{Results}

Excess RA induced miR-27b-3p expression in early differentiation stage of $\mathrm{C2C12}$

For investigation of RA influence on the expression of miR-27b- 


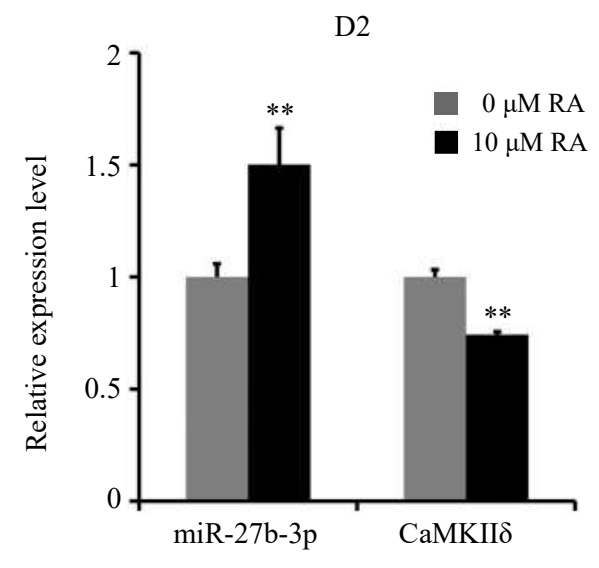

Figure 2. Real-time PCR analyzed the mRNA expression of miR-27b-3p was and CaMKII $\delta$ in cells cultured in DM supplemented with or without $10 \mu \mathrm{M}$ RA for 2 days. Results were presented as means $\pm \mathrm{SD}$ of triplicate experiments. $* \mathrm{P}<0.05, * * \mathrm{P}<0.01$.

$3 p$ and CaMKII $\delta$, cells treated by $10 \mu \mathrm{M}$ RA for 2 days after myogenic differentiation induction were collected. Immunofluorescence staining indicated that CaMKII $\delta$ protein reduced and myosin reduction implied the inhibition of early differentiation of $\mathrm{C} 2 \mathrm{C} 12$ with excess RA addition (Fig. 1). qRT-PCR results showed that the transcription of miR-27b-3p was up-regulated whereas the CaMKII $\delta$ mRNA was inhibited (Fig. 2).

\section{miR-27b-3p directly targets 3' CaMKIISUTR and inhibits CaMKIID expression}

Our recently studies showed that the expression of miR-27b-3p was up-regulated significantly both in E14.5 mouse tongue and in $\mathrm{C} 2 \mathrm{C} 12$ cells cultured in DM from the 2 nd day ${ }^{15)}$ in condition of excess RA then control. In the meantime, the expression of CaMKII $\delta$ was decreased at the same stages ${ }^{14)}$. Bioinformation analysis using TargetScan, MiRanda, and MicroCosm predicted that a conserve complementary site in both the 3'UTRof CamkIII (Fig. 3A) and DTNA ${ }^{15)}$ for the seed sequence of miR-27b-3p. We also found another miRNA, miR-31-5p could also complementary match with the 3 ' UTR of CamkII ${ }^{14)}$, but the effects of both miR-27b-3p targeted to DTNA and miR-31-5p to CaMKII $\delta$ were mainly displayed in the late stages of $\mathrm{C} 2 \mathrm{C} 12$ myogenic differentiation $^{14,15)}$. So, whether miR-27b-3p targeted CaMKII $\delta$ affected

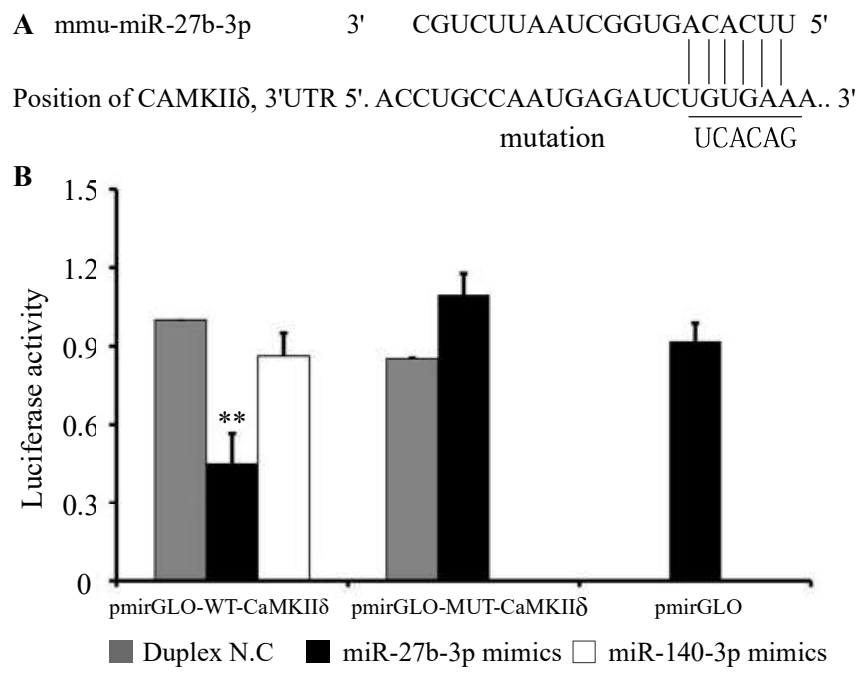

Figure 3. The vector sequences of pmirGLO- CaMKII $\delta$-WT (WT) and pmirGLO- CaMKII $\delta$-MUT (MUT) were designed for luciferase activity assay according to the TargetScan predicted results (A). Luciferase activities were detected after the plasmids of WT, MUT and pmirGLO were co-transfected into $293 \mathrm{~T}$ cells with Duplex NC, miR-27b-3p and miR-140-3p mimic, respectively. miR-27b-3p but not Duplex NC or miR140-3p, significantly inhibited the luciferase activities of the WT; by comparison, it didn't affect the activities of MUT and empty pmirGLO (B). Results were presented as means $\pm \mathrm{SD}$ of triplicate experiments. ${ }^{*} \mathrm{P}<0.05$, $* * \mathrm{P}<0.01$.

the other processes of myogenesis attracted our attention. Combine the above results (Fig. 1 and 2), RA induced the increase of miR-27b$3 p$ and decrease of CaMKII $\delta$ at early stage of $\mathrm{C} 2 \mathrm{C} 12$ differentiation. For further confirmation of the predict result between miR-27b-3p and CaMKII $\delta$ by Bioinformation analysis, luciferase reporter assay was conducted in $293 \mathrm{~T}$ cells. The results indicated that luciferase activity was significantly repressed in the cells co-transfected with the miR27b-3p mimic and pmirGLO-CaMKII $\delta$-WT compared with Duplex $\mathrm{NC}$ and miR-140-3p groups, while miR-27b-3p mimic displayed no effect on the luciferase activity of pmirGLO-CaMKII $\delta$-Mut as well as the Duplex NC (Fig. 3B). Moreover, the impact of miR-27b-3p on CaMKII $\delta$ expression was detected at the meantime, and the qRT-PCR revealed that miR-27b-3p suppressed the mRNA levels of CaMKII $\delta$
A

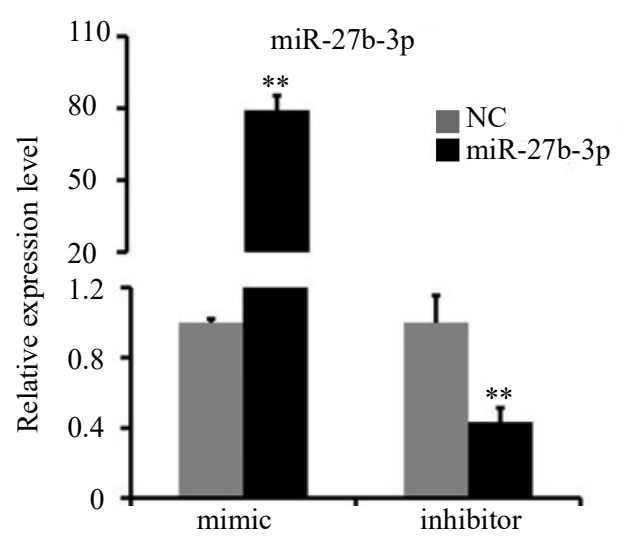

B

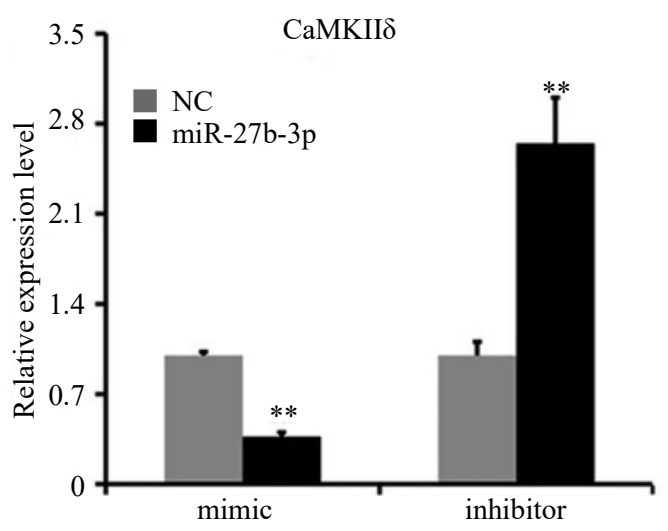

Figure 4. Real-time PCR analyzed the mRNA expression of miR-27b-3p (A) and CaMKII $\delta$ (B) after C2C12 cells were tansfected with miR-27b$3 \mathrm{p}$ mimic or inhibitor. Results were presented as means $\pm \mathrm{SD}$ of triplicate experiments. ${ }^{*} \mathrm{P}<0.05,{ }^{*} \mathrm{P}<0.01$. 
Bo Liu et al.: miR-27b-3p Targeting CaMKII $\delta$ Rescued Excess RA Induced Abnormal Myogenic Differentiation

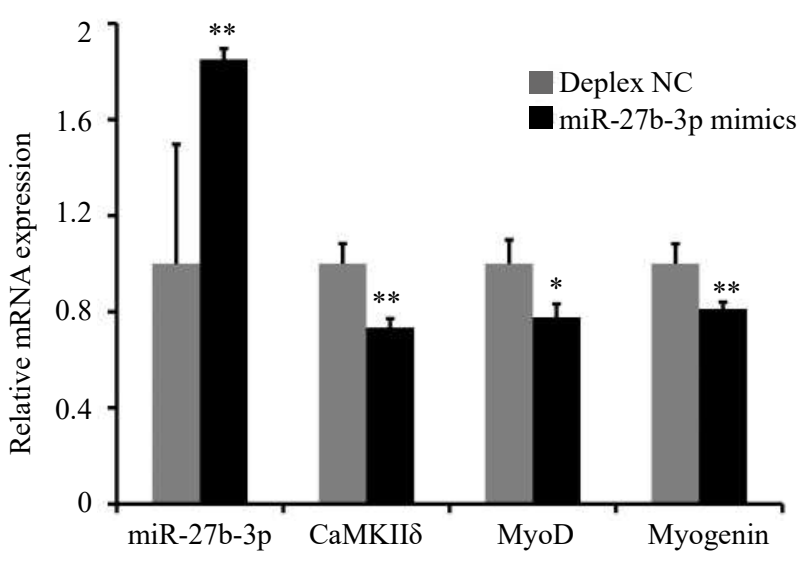

Figure 5. Real-time PCR analyzed the mRNA expression of miR-27b$3 p$, CaMKII $\delta$, MyoD and Myogenin in $\mathrm{C} 2 \mathrm{C} 12$ cells after transfected with miR-27b-3p mimic for 2 days in DM. Results were presented as means \pm $\mathrm{SD}$ of triplicate experiments. $* \mathrm{P}<0.05, * * \mathrm{P}<0.01$

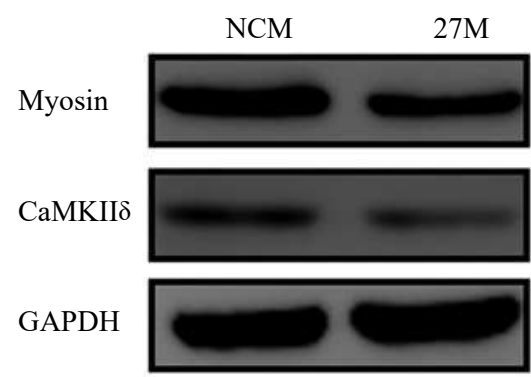

Figure 6. Western blot analyzed Myosin and CaMKII $\delta$ protein expression of $\mathrm{C} 2 \mathrm{C} 12$ cells after transfected with miR-27b-3p mimic for 2 days in DM.
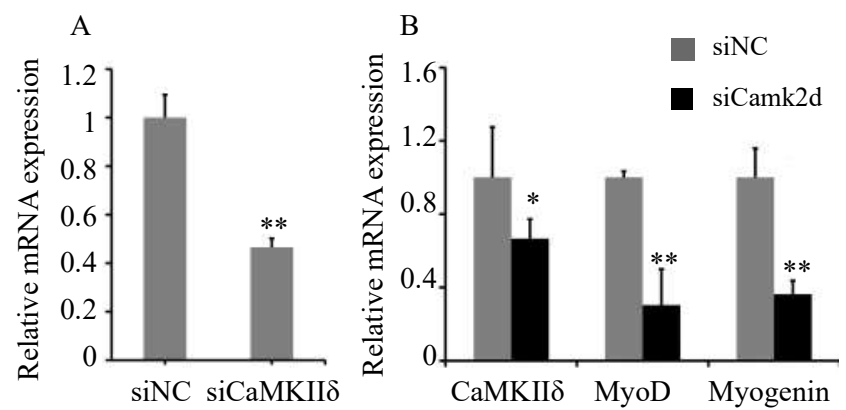

Figure 8. Real-time PCR analyzed the knock down efficiency of siCaMKII $\delta$ and the mRNA expression of CaMKII $\delta$, MyoD and Myogenin in $\mathrm{C} 2 \mathrm{C} 12$ cells after transfected with siCaMKII $\delta$ for 2 days in DM. Results were presented as means $\pm \mathrm{SD}$ of triplicate experiments. $* \mathrm{P}<0.05, * * \mathrm{P}<$ 0.01 .

A

siNC $\quad$ siCaMKII $\delta$

CaMKII

GAPDH

B
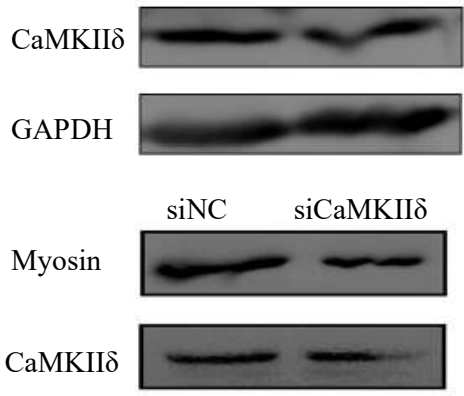

GAPDH

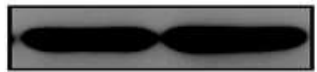

Figure 9. Western blot analyzed silencing efficiency of siCaMKII $\delta$ (A) and Myosin and CaMKII $\delta$ protein expression of $\mathrm{C} 2 \mathrm{C} 12$ cells after transfected with siCaMKII $\delta$ for 2 days in DM (B).

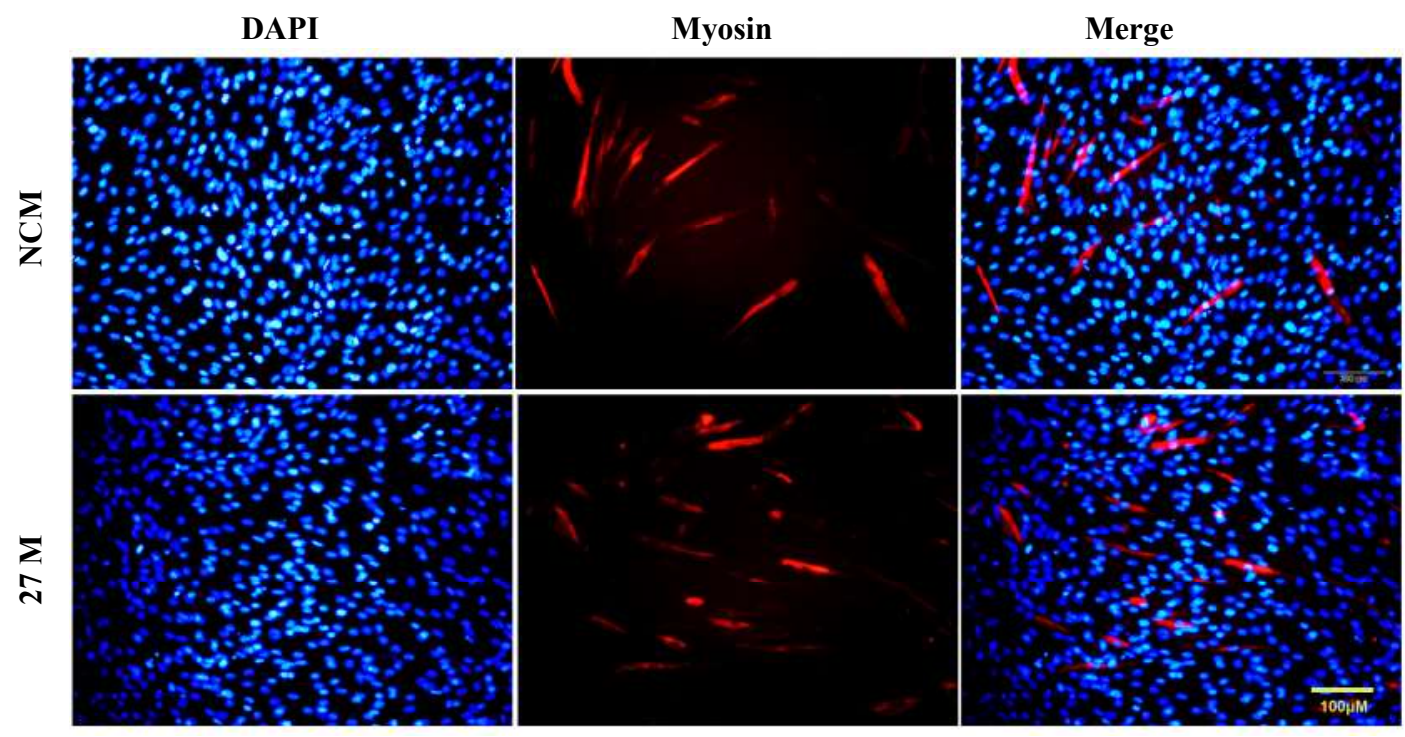

Figure 7. Immunofluorenscence staining analyzed the myosin expression in C2C12 cells transfectied with miR-27b-3p mimic for 2 days in DM. Bar $=100 \mu \mathrm{m}$ 

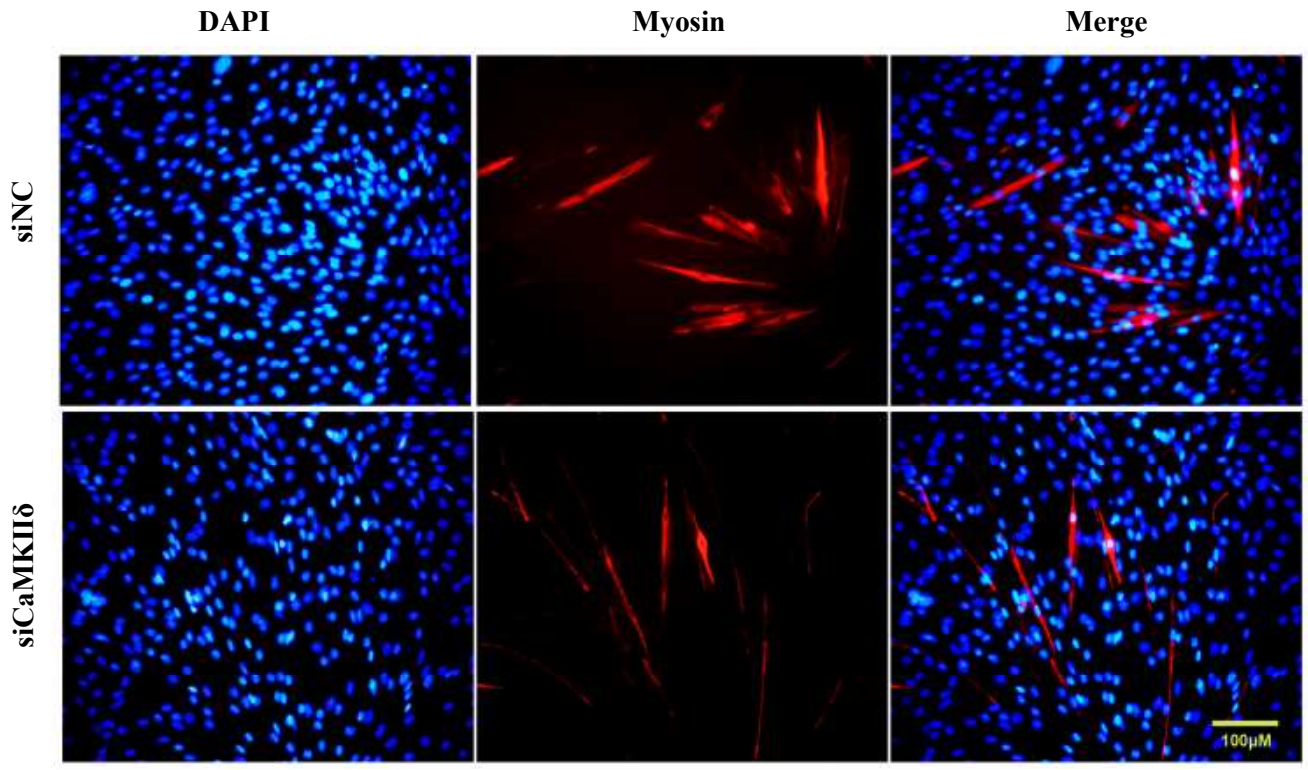

Figure 10. Immunofluorenscence staining analyzed the myosin expression in $\mathrm{C} 2 \mathrm{C} 12$ cells transfectied with siCaMKII $\delta$ for 2 days in DM. $\mathrm{Bar}=100 \mu \mathrm{m}$.
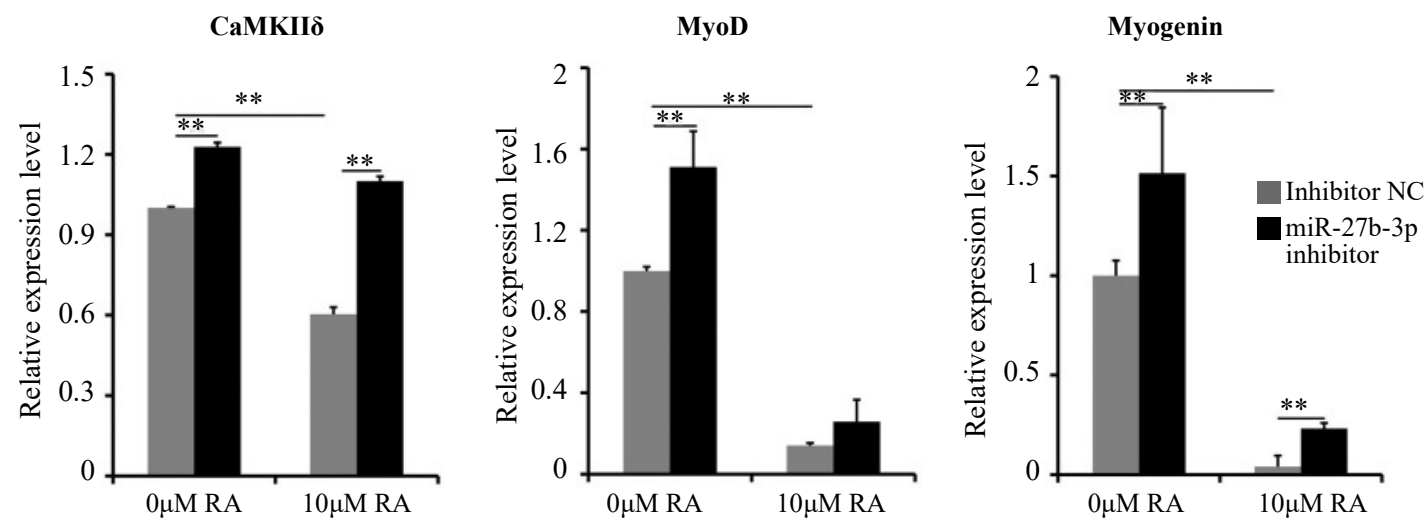

Figure 11. Real-time PCR analyzed the mRNA expression of CaMKII $\delta$, MyoD and Myogenin in C2C12 cells transfected with miR-27b-3p inhibitor with or without RA in DM for 2 days. Results were presented as means $\pm \mathrm{SD}$ of triplicate experiments. ${ }^{*} \mathrm{P}<0.05,{ }^{*} \mathrm{P}<0.01$.

(Fig. 4A and B). In summary, these results demonstrated that miR-27b$3 p$ directly complementary with the 3'UTRof CaMKII $\delta$ and repressed CaMKIII expression.

\section{miR-27b-3p over-expression repressed early differentiation of C2C12}

As shown in Fig. 1 and 2, our results indicated that miR-27b-3p and CaMKIII expression was up- and down-regulated in RA treated cells on D2 separately. Then we determined whether miR-27b-3p overexpression could suppress the early differentiation of $\mathrm{C} 2 \mathrm{C} 12$ significantly. miR-27b-3p mimic and Duplex NC were transfected into $\mathrm{C} 2 \mathrm{C} 12$ cells at the beginning of differentiation. As shown in Fig. 5 after 2 days of transfection, the miR-27b-3p expression increased prominently compared with the cells transfected with Duplex NC. However, the CaMKII $\delta$ expression as well as two myogenic regulatory factors MyoD and Myogenin, was repressed significantly (Fig. 5). Western blot results also indicated that the protein level of CaMKII was decreased too (Fig. 6). To confirm the impaired effects of miR-27b$3 p$ on the early differentiation of $\mathrm{C} 2 \mathrm{C} 12$ cells, the myosin expression and myotube formation were detected by immunofluorescence staining, results showed that the number and length of myosin positive myotubes were decreased by miR-27b-3p mimic (Fig. 7). Taken together, these results demonstrated that miR-27b-3p mimic decreased CaMKII $\delta$ expression and inhibited early differentiation of $\mathrm{C} 2 \mathrm{C} 12$.

\section{Knockdown of CaMKIIS impaired the early differentiation of C2C12}

In order to further explore whether the inhibition of CaMKII $\delta$ expression in early differentiation stage of $\mathrm{C} 2 \mathrm{C} 12$ was the main cause for the disruption of myogenic differentiation, cells were transfected with siCaMKII $\delta$ to knockdown CaMKII $\delta$ expression during differentiation process. As expected, after CaMKII $\delta$ expression was knockdown (Fig. 8), the transcriptions of CaMKII $\delta$, MyoD and Myogenin were inhibited significantly after 2 days cultured in DM (Fig. 8). Meanwhile, the remarkably decreased protein levels of myosin and CaMKII $\delta$ indicating by western blot also implicated an inhibition on early differentiation of $\mathrm{C} 2 \mathrm{C} 12$ by siCaMKIII (Fig. 9). The immunofluorescence staining further confirmed the reduction of myosin in early differentiating $\mathrm{C} 2 \mathrm{C} 12$ induced by siCaMKII $\delta$ (Fig. 10). These results showed that interference on CaMKII $\delta$ was sufficient to mediate the excess RA repression on early differentiation of $\mathrm{C} 2 \mathrm{C} 12$ cells.

Inhibition of miR-27b-3p partly abrogates early differentiation damage of $\mathrm{C} 2 \mathrm{C} 12$ induced by excess $\mathrm{RA}$ 


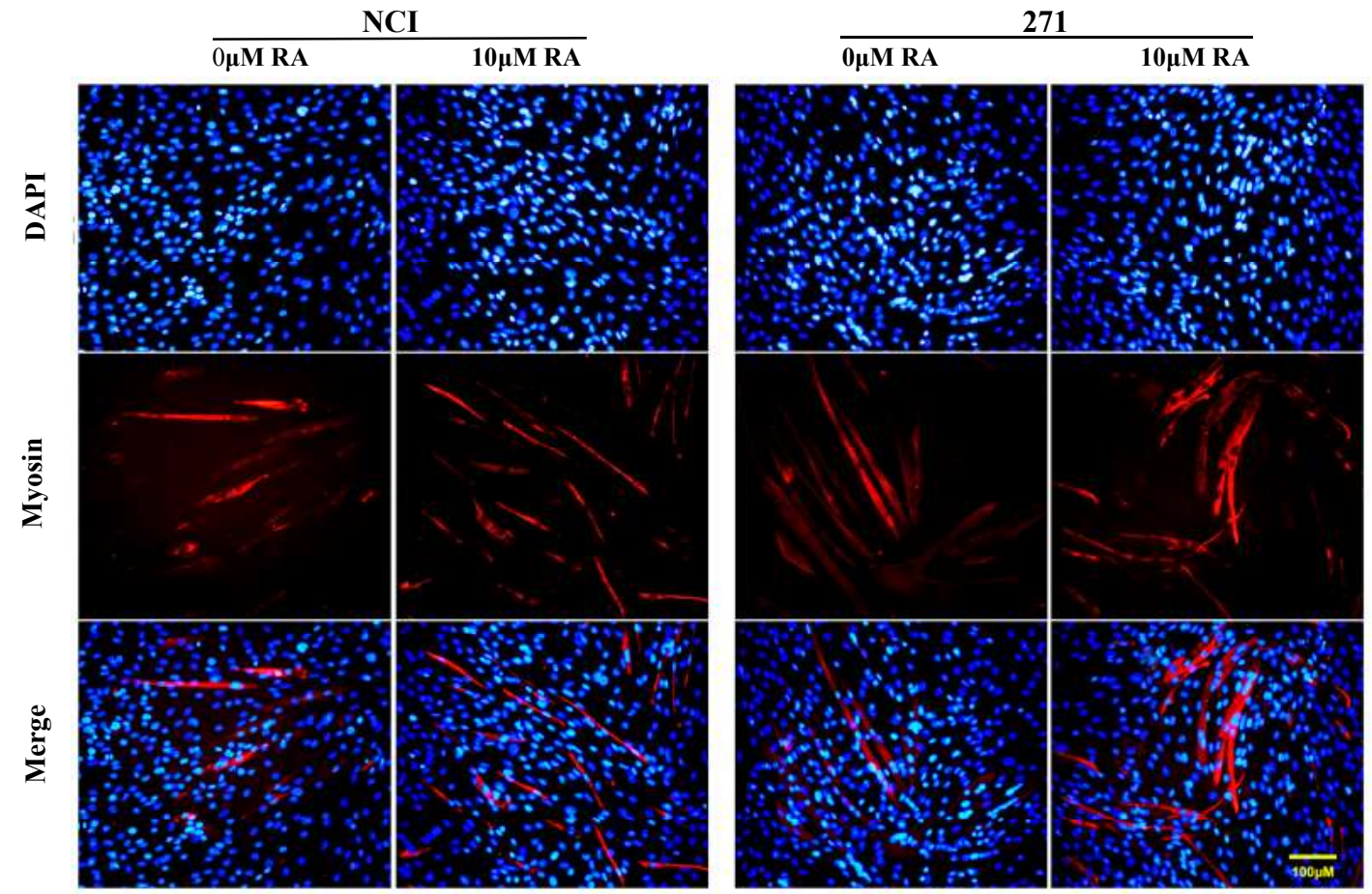

Figure 12. Immunofluorenscence staining analyzed the myosin expression in C2C12 cells transfectied with miR-27b-3p inhibitor with or without $10 \mu \mathrm{M}$ RA in DM for 2 days. Bar $=100 \mu \mathrm{m}$.

To investigate whether the RA induced inhibition of $\mathrm{C} 2 \mathrm{C} 12$ early differentiation was miR-27b-3p dependent, cells were transfected with miR-27b-3p inhibitor when treated with $10 \mu \mathrm{M}$ RA. The mRNA levels of CaMKII $\delta$ and Myogenin were significantly increased by miR-27b-3p inhibitor with or without RA (Fig. 11), but the MyoD mRNA increase was not significant in presence of RA (Fig. 11), implying that Myogenin was the main target of miR-27b-3p inhibitor when abrogated the disruption of excess RA. Immunofluorence staining results of myosin further confirm the rescue by miR-27b-3p inhibitor (Fig. 12). Therefore, miR-27b-3p inhibitor could partly rescued $\mathrm{C} 2 \mathrm{C} 12$ early differentiation caused by RA especially through regulating Myogenin expression.

\section{Discussion}

miRNAs are small non-coding RNAs which regulate gene expression at post-transcription level. miRNAs have been proved involved in skeletal myogenesis by interacting with MRFs and miRNA dysregulation are closely associated with muscular pathology ${ }^{16)}$. miR$27 \mathrm{~b}$ has been shown to inhibit adipocyte differentiation by repressing PPAR $\gamma$ and CREB expression, while TNF- $\alpha$ could stimulate miR-27 expression via NF-kB pathway ${ }^{17}$. Zhang $^{18)}$ and his colleagues proved that during the porcine myoblast differentiation, miR-27a expression was rebounded back after a transient decrease at early differentiation stage. Furthermore, they found that miR-27a inhibited the Myogenin expression as well as the $\mathrm{Akt} / \mathrm{FoxO} 1$ pathway in this process. In our latest study ${ }^{15)}$, we found that miR-27b-3p expression pattern was very similar with the miR-27a expression in the above report. In this study, we identified that when CaMKII $\delta$ was up-regulated by miR-27b-3p inhibitor in cells treated with $10 \mu \mathrm{M}$ RA, the Myogenin expression was enhanced more than MyoD, which implied that the rescue results of miR-27b-3p targeted CaMKII $\delta$ in RA induced early myogeic differentiation damage was mainly through Myogenin. Thus, if there is a direct interaction between CaMKIII and Myogenin need our further detection.
Ubiquitously expressed and multifunctional CaMKII is the main CaMK in skeletal muscle and its four isoforms $(-\alpha,-\beta,-\gamma$, and $-\delta)$ distribute in a cell-type specificity manner ${ }^{19)}$. Increased sarcoplasmic CaMKII content affected the skeletal muscle phenotype in vivo ${ }^{20)}$. Recently studies showed that the expression of CaMKII $\delta$ was regulated by certain non-coding RNAs in pathologic processes. For example, miR-145 targeted CaMKII $\delta$ directly to regulate reactive oxygen species (ROS)-induced cardiomyocytes apoptosis ${ }^{21)}$. CaMKII $\delta$ plays key roles in vascular smooth muscle (VSM) by regulating proliferation, migration and injury-induced vascular neointima formation, and it was reported that miR-30 family members could inhibit neointimal hyperplasia by directly regulating CaMKII $\delta$ expression after vascular injury ${ }^{22)}$. In this study, based on the bioinformatics analysis we speculated that CaMKII $\delta$ might be a target of miR-27b-3p in myoblasts. As expected, gain- and loss-of function experiments validated miR-27b-3p negatively regulated CaMKII $\delta$ expression and luciferase reporter assay demonstrated that CaMKII $\delta$ was a target of miR-27b-3p.

CaMKII plays critical role in the $\mathrm{Wnt} / \mathrm{Ca}^{2+}$ pathway, we previous found that Wnt/CaMKII $\delta$ pathway was involved in excess RA-induced abnormal tongue development of embryonic mice ${ }^{13)}$. To explore the molecular mechanism for this, we subsequently did miRNA microarray and identified the dysregulated miRNAs in RA-induced abnormal tongue ${ }^{15)}$ and studies in this paper, we found that CaMKII $\delta$ was a downstream target of miR-27b-3p. Furthermore, our latest studies also indicated that miR-27b-3p and miR-31-5p could target DTNA $^{15)}$ and CaMKII $\delta^{14)}$ respectively at late myogenic differentiation stages, implying that miR-27b-3p controls different targets and CaMKII $\delta$ could be regulated by numbers of miRNAs at different stages during myogenic differentiation process. These were consistent with the characters and complex regulatory network of miRNAs. More importantly, miR-27b-3p could target CaMKII $\delta$ and DTNA simultaneously although at different stages, which suggested a potential 179 
J.Hard Tissue Biology Vol. 27(3): 173-180, 2018

myoblasts and thus further investigations are needed to confirm.

In conclusion, the downregulation of CaMKII $\delta$ in early myogenic differentiation stage demonstrated the involvement of this molecule in the progression of RA induced abnormal myogenic differentiation. The results of luciferase reporter assay indicated that miR-27b-3p could directly regulate CaMKII $\delta$ expression to exert its antagonism for excess RA in myogenic differentiation. All these indicated that miR-27b-3p and CaMKII $\delta$ may act as biomarkers and potential therapeutic targets for RA induced muscle deformity.

\section{Acknowledgement}

The work of this study was supported by National Natural Science Foundation of China (No: 81570962) and the National Natural Science Foundation of China for Youth Scientists (No: 81500827).

\section{Competing Interests}

The authors declare there are no COI regarding the publication of this paper.

\section{References}

1. Duprey P and Lesens C. Control of skeletal muscle- specific transcription: involvement of paired homeodomain and MADS domain transcription factors. Int J Dev Biol 38: 591-604, 1994

2. Lee GS, Kochhar DM and Collins MD. Retinoid-induced limb malformations. Curr Pharm Des 10: 2657-2699, 2004

3. Xiao J, Cong W, Wang R, Wang B, Wang F, Zhu E-x, Hu H, Katase $\mathrm{N}$ and Nagatsuka $\mathrm{H}$. The study of palatal cell proliferation and apoptosis in retinoic acidinduced mouse cleft palate varied with different developmental stage. J Hard Tissue Biol 6:125-130, 2007

4. Fabian MR, Sonenberg N and Filipowicz W. Regulation of mRNA translation and stability by microRNAs. Annu Rev Biochem 79: 351-379, 2010

5. Lagos-Quintana M, Rauhut R, Yalcin A, Meyer J, Lendeckel W and Tuschl T. Identification of tissue- specific microRNAs from mouse. Curr Biol 12: 735-739, 2002

6. McCarthy JJ. MicroRNA-206: the skeletal muscle-specific myomiR. Biochim Biophys Acta 1779: 682-691, 2008

7. Zhao Y and Srivastava D. A developmental view of microRNA function. Trends Biochem Sci 32: 189-197, 2007

8. Marzi MJ, Puggioni EM, Dall'Olio V, Bucci G, Bernard L, Bianchi F, Crescenzi M, Di Fiore PP and Nicassio F. Differentiationassociated micro-RNAs antagonize the Rb-E2F pathway to restrict proliferation. J Cell Biol 199: 77-95, 2012

9. Lee JJ, Drakaki A, Iliopoulos D and Struhl K. MiR-27b targets PPAR $\gamma$ to inhibit growth, tumor progression, and the inflammatory response in neuroblastoma cells. Oncogene 31: 3818-3825, 2012

10. Crist CG, Montarras D, Pallafacchina G, Rocancourt D, Cumano A, Conway SJ and Buckingham M. Muscle stem cell behavior is modified by microRNA-27 regulation of Pax 3 expression. Proc
Natl Acad Sci U S A 106: 13383-13387, 2009

11. Allen DL and Loh AS. Posttranscriptional mechanisms involving microRNA-27a and $\mathrm{b}$ contribute to fast-specific and glucocorticoidmediated myostatin expression in skeletal muscle. Am J Physiol Cell Physiol 300: C124-C137, 2011

12. Minetti GC, Feige JN, Bombard F, Heier A, Morvan F, Nürnberg B, Leiss V, Birnbaumer L, Glass DJ and Fornaro M. Gai2 signaling is required for skeletal muscle growth, regeneration, and satellite cell proliferation and differentiation. Mol Cell Biol 34: 619-630, 2014

13. Cong W, Liu B, Liu S, Sun M, Liu H, Yang Y, Wang R and Xiao J. Implications of the Wnt5a/CaMKII pathway in retinoic acidinduced myogenic tongue abnormalities of developing mice. Sci Rep 4: 6082, 2014

14. Liu B, Liu C, Cong W, Li N, Zhou N, Tang Y, Wei C, Bai H, Zhang $\mathrm{Y}$ and Xiao J. Retinoid acid-induced microRNA-31-5p suppresses myogenic proliferation and differentiation by targeting CamkII $\delta$. Skelet Muscle 7: 8, 2017

15. Li N, Tang Y, Liu B, Cong W, Liu C and Xiao J. Retinoid acidinduced microRNA-27b-3p impairs $\mathrm{C} 2 \mathrm{C} 12$ myoblast proliferation and differentiation by suppressing $\alpha$-dystrobrevin. Exp Cell Res 350: 301-311, 2017

16. Goljanek-Whysall K, Sweetman D and Münsterberg AE. microRNAs in skeletal muscle differentiation and disease. Clin Sci (Lond) 123: 611-625, 2012

17. Zhu Y, Zhang X, Ding X, Wang H, Chen X, Zhao H, Jia Y, Liu S and Liu Y. miR-27 inhibits adipocyte differentiation via suppressing CREB expression. Acta Biochim Biophys Sin (Shanghai) 46: 590596, 2014

18. Zhang S, Chen X, Huang Z, Chen D, Yu B, He J, Zheng P, Yu J, Luo J, Luo Y and Chen H. Effects of microRNA-27a on myogenin expression and $\mathrm{Akt} / \mathrm{FoxO} 1$ signal pathway during porcine myoblast differentiation. Anim Biotechnol 11: 1-7, 2017

19. Takemoto-Kimura S, Suzuki K, Horigane SI, Kamijo S, Inoue M, Sakamoto M, Fujii H and Bito H. Calmodulin kinases: essential regulators in health and disease. J Neurochem 141: 808-818, 2017

20. Eilers W, Jaspers RT, de Haan A, Ferrié C, Valdivieso P and Flück M. CaMKII content affects contractile, but not mitochondrial, characteristics in regenerating skeletal muscle. BMC Physiol 14: 7, 2014

21. Cha MJ, Jang JK, Ham O, Song BW, Lee SY, Lee CY, Park JH, Lee J, Seo HH, Choi E, Jeon WM, Hwang HJ, Shin HT, Choi E and Hwang KC. MicroRNA-145 suppresses ROS-induced $\mathrm{Ca}^{2+}$ overload of cardiomyocytes by targeting CaMKII $\delta$. Biochem Biophys Res Commun 435: 720-726, 2013

22. Liu YF, Spinelli A, Sun LY, Jiang M, Singer DV, Ginnan R, Saddouk FZ, Van Riper D and Singer HA. MicroRNA-30 inhibits neointimal hyperplasia by targeting $\mathrm{Ca}(2+) /$ calmodulin-dependent

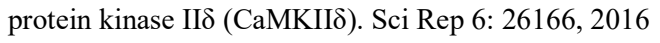

